\title{
Wolfgang Hecker Vorbild New York? \\ Zur aktuellen Debatte über eine neue Sicherheits- und Kriminalpolitik
}

\begin{abstract}
Die aktuelle Debatte zur Sicherheits- und Kriminalpolitik in der BRD befaßt sich zunehmend mit dem "Modell New York *U Über die Fachdebatte hinaus ist das Thema auch Gegenstand zablreicher Medienberichte. Der Spiegel widmete ihm einen Titelbericht.' Vertreter von Polizei-und Ordnungsbehörden in der BRD reisen nach New York, um Erfolge und Übertragbarkeit des Modells New York zu prüfen. Der vorliegende Beitrag befaßt sich mit den kriminologischen Prämissen (Theorie der broken windows), der praktischen Umsetzung und rechtlichen Problematik dieses Modells. Vor dem Hintergrund der Debatte über das Modell New York werden die Perspektiven kommunaler Prävention in der BRD erörtert.
\end{abstract}

Die Red.

\section{Die Theorie der broken windows}

In der aktucllen Debatte über eine neue Sicherheits- und Kriminalpolitik kommt der Theorie der "broken windows " (zcrbrochenen Fenster) eine entscheidende Bedeutung zu. Populär ausformuliert wurde diese Theorie in einem Bcitrag von Wilson und Kelling, der erstmalig bereits 1982 veröffentlicht wurde." Nicht zufällig fand dieser Beitrag in den leczten Jahren ein großes Medienecho. Die Art und Wcise sciner Abfassung weist ihn als populärwissenschaftlichen Beitrag aus, der sich gezielt an die allgemeine Öffentlichkeit im Sinne cincr "newsmaking criminology « wendet. ${ }^{3}$

Den Ausgangspunkt der Überlegungen von Wilson und Kelling bilder die Debatte zur Frage eines verstärkten Einsatzes von Fußstreifen der Polizei im Staat New Jersey Mitte der yoer Jahre. Ein entsprechendes, vom Staat New Jersey gefördertes Programm wurde sehr kontrovers diskutiert. Zum Teil wurde der Einfluß verstärkter allgemeiner polizeilicher Präsenz auf die Kriminalitätsentwicklung in Frage gestellt, andere Bedenken betrafen u. a. die Immobilität von Fußstreifen. Zentraler Gegenstand der Debatte war die Frage, ob sich polizeiliche Kräfte weiter auf die Verfolgung vor allem von schwerer Kriminalität konzentricren sollten. Mit dem Fußstreifenprojekt wurde ein neuer Weg beschritten. An Stelle der bloßen Verfolgung von Straftaten wurde die Polizei aus dem Streifenwagen herausgcholt und zur

1 Spiegél 28/1997.

2 J.W. Wilson/G. L. Kellıng. The police and neighborhoud safecy: Broken Windows, in: Atlantic Monthly, Marz 1982, S. 29-39, im folgenden zituert nach der deutschen Überseczung von B. Paul, Polizei und Nachbarschafissicherheit: Zerbrochene Fenster, in: Kriminologisches Journal 2/1996, $121-136$.

3 Dazu spezicll sowie zum allgemeinen Entsiehungszusammenhang in Rahmen der kriminologischen Debasce in den USA, F. Sack, Krminologie - popular gemach,, in: Krimunologisches Journal 2/1997, 116-120, und H. Hess, New York zieht die Lehren aus den zerbrochenen Fensterscheiben, in: Krimınologisches Journal 3/1997, 179-190. 
Gewährleistung einer verstärkten Präscnz in den Wohngebietcn eingesctzt. Eine Auswertung des Fußstreifen-Projekts in New Jersey ergab, daß sich die Kriminalitätsrate zwar nicht verringert hatce, das subjektive Sicherheitsgefühl der Bewohner aber deutlich gestiegen war. ${ }^{+}$

In Auseinandersetzung mit diesem Ergebnis formulieren Wilson und Kelling die erste, für das Verständnis ihrer Konzeption von Prävention entscheidende These: Auch wenn die Kriminalitätsraten nicht gesunken waren, wurde das Leben für die Wohnbevölkerung auf Grund der Fußstreifen insgesamt dennoch "sicherer «. Zwar fürchteten sich Menschen in crster Linie vor Kriminalität, dabei dürfe aber cine andere wichtige Quelle für Angst nicht übersehen werden:

... »die Angst, von unangenehmen Personen beläsrigt zu werden. Es müssen nicht unbedingt gewalttätige oder kriminelle Personen sein, sondern solche mit schlecbtem Ruf, lärmender Aufdringlich-oder Unberechenbarkeit: Bettler, Betrunkene, Süchtige, randalierende Jugendliche, Prostituierte, Herumhängende und psychisch Kranke. «s

Vor allem für ältere Menschen könne die Konfrontation mit cinem lärmenden Jugendlichen oder eincm betrunkenen Bettler cbenso mit Angst besetzt sein wie dic Begegnung mit einem wirklichen Raubtäter. Allgemein zitieren Wilson und Kelling in diesem Zusammenhang verschiedene Untersuchungen zur subjektiven Bedeutung gefährlicher Orte. Danach wurden häufig von Bewohnern solche Orte als gefährlich angesehen, an denen ernsthafte Gcfahren objektiv nicht aufgetreten waren (Treffpunkte von Jugendlichen usw.). Wilson und Kelling verweisen in diesem Zusammenhang auch auf die Bedeutung von Graffiti für Nutzer von Verkehrsmitteln. Mit derartigen Graffiti werde signalisiert, daß es sich um unkontrollierte und unkontrollierbare Räume handele. ${ }^{6}$

Nach Auffassung von Wilson und Kelling ist es letzrlich ohne Bedeutung, in welchem Anteil berechtigre Angst vor realer Kriminalität den Ruf der Bewohner nach einem Einschreiten der Ordnungskräfte begründet, und in welchem Ausmaß es um cin bloßes Unwohlsein wegen der »Unordnung " auf der Straße geht. Ein weitreichendes Einschreiten sei in beiden Fällen gleichermaßen berechtigt. Zusrimmend verweisen Kelling und Wilson dabei auf die Entwicklung einer entsprechenden Praxis im kommunalen Bereich zum Zeitpunkt ihrer Untersuchung. Beispielsartig wird die Praxis der Duldung von Alkoholkonsum allenfalls in Seitenstraßen, das Vorgehen gegen Betteln an Bushaltestellen usw. geschildert. Wer als Fremder keine befriedigende Auskunft über seinen Aufenthaltsgrund bzw. seine persönlichen Lebensverhältnisse geben konnte, wurde ggfls. zum Verlassen des Gebiets aufgefordert. Bei Verstößen erfolgten z. T. Verhaftungen u. a. wegen Landstreicherei?

Bemerkenswert an der von Wilson und Kelling beschriebenen Praxis einer von den subjektiven Vorstellungen der jeweiligen community formulierten kommunalen Ordnungspolitik ist die weitgehend rechtliche Ungebundenheit. Grundrechte oder sonstige Rechtsgrundsätze treten fast völlig zurück hinter den subjektiven Ordnungsvorstellungen der jewciligen community. Wilson und Kelling betonen ausdrücklich selbst, daß ein relevanter Teil der von ihnen beobachteten polizcilichen Praxis einer strengen rechtlichen Überprüfung wohl kaum standhalten könne. ${ }^{8}$ Die rechtliche Problematik wird in den Überlcgungen von Wilson und Kelling aber nicht weiter thematisiert. Den Schwerpunkt ihrer Ưberlegungen bildet allein die These einer kriminalitätsverhütenden Wirkung dieser Praxis (sowie des positiven Echos bei der Bevölkerung).

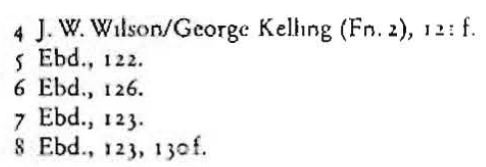


Zentraler Gegenstand der Überlegungen von Wilson und Kelling ist der Zusammenhang zwischen "Unordnung "im öffentlichen Raum und Kriminalität. Dazu lautet die Grund these, daß Unordnung und Kriminalität normalerweise unentwirrbar niceinander verbunden seien. Die Annahme einer derart ursächlichen Abfolge zwischen Unordnung und Kriminalität wird insbesondcre anhand des vielfach zitierten Beispiels der broken windows erläutert. Ein nicht wieder in Stand gesetztes zerbrochenes Fenster stellt danach ein Zeichen dafür dar, daß an dem entsprechenden Ort auf Verstöße keinc Rcaktion erfolgt. Es können also auch weitere Fenster zerstört werden bzw. sonstige Verstöße und Straftaten begangen werden. Wilson und Kelling stützen sich dabei auf die Ergebnisse entsprechender Experimente des StanfordPsychologen Zimbardo aus den 6oer Jahren. ${ }^{9}$ Auf der Grundlage dieser Überlegungen entwickeln Wilson und Kelling ein allgemcines Entwicklungsmodell von Kriminalität, das seinen Anfang bei kleinen Verstößen gegen Recht und Ordnung nimmt, sich in zunchmendem Vandalismus, Verwahrlosung, Alkoholismus und Betteln usw. im öffentlichen Raum fortsetzt, und schlicßlich häufig in ernstzunehmende Kriminalität einmündet. Als entscheidende Ursache dieser Abfolge wird Gleichgültigkeit gegenüber Verstößen gegen Recht und Ordnung vor allem in der Frühphase der Entwicklung ausgemacht. Damit setze ein Zusammenbruch der informellen Kontrolle ("community controls«) ein, die Wilson und Kelling als entscheidende Grundlage stabiler Nachbarschaft betrachten. ${ }^{10}$

Die hier vorliegende Problematik thematisieren Wilson und Kelling als allgemeinen Prozeß "städtischen Verfalls", der zwar vorrangig in bestimmten sozialen Brennpunkten auftretc, potentiell aber überall einsetzen könne, wo Sicherheit gervährleistende stabile Strukturen verloren gegangen seien. Während traditionclle Gemeinden durch eine relativ feste Einwohnerschaft gekcnnzeichnet seien, die sich entschlossen in ihrem Gemeindegebiet gegen Rechtsverletzungen zur Wchr sctzten, fehle es in der modernen Stadt an derartigen Selbstregulierungsmcchanismen. An die Stelle entschlossener Nachbarschaften seien hier häufig Gilcichgültigkeit oder Wegzug derjenigen, die es sich leisten können, getreten. Daneben sei die unmittelbarc polizeiliche Präscnz in den Gemeinden immer mchr reduzicrt worden. Ein vercngtes Modell der Verbrechensbekämpfung und Festnahme von Tätern habe zur Entwertung der eigentlichen polizeilichen Aufgabe geführt. Vergessen worden sei der Zusammenhang von Ordnungserhaltung und Verbrechensbekämpfung. "

Dic Neubegründung eines adäquaten Konzepts öffentlicher Sicherheit setzt nach Wilson und Kelling voraus, daß die informelle Kontrolle über das Gemeindegebiet durch seine Bcrvohner wiedererlangt wird. Dazu bedürfe es ausreichender polizeilicher Unterstützung. Für relcvant wird aber nicht allein eine verstärkte personelle Ausstattung der Polizei erachtet. ${ }^{12}$ Entscheidend sei vor allem ein Umdenken in der Rollenwahrnehmung. An die Stelle der Verfolgung begangener Straftaten - zudem häufig konzentriert auf schwere Straftaten - müsse wieder eine umfassende, vor allem präventive Verantwortung für die öffentliche Sicherheit und Ordnung trctcn. Hier angesiedelte Bedürfnisse der Gemeinschaft ("community needs") seien im Modell des überkommenen liberalen Rechtsstaats nicht hinreichend berücksichtigt worden. Das durch die Koordinaten individuelle Rechte, zu bcachtende Vorschriften und die individuelle Verfolgung von Rechtsverstößen bestimmte Modell trage den

9 Ebd., 124.

10 Ebd., $125 \mathrm{ff}$.

11 E.bd., 127 f., 130.

12 Zum Verstandnis des Beitrags und der allgemennen Debatte in den USA (auch im Hinblick auf Vergleiche mit der BRD) ist die allgemein drastische Ausdunnung der personellen Kapazıtaten der Polizei in den USA im Entstehungszeitpunkt des Beitrags von Wilson und Kelling von Bedeutung. 
Verlusten, die im Zuge des allgemeinen Verfalls der Städtc dem Gemeinwesen erwüchsen, nicht mehr hinreichend Rechnung. Es bedürfe somit einer neuen Gewichtung des Verhältnisses von Schutz des Individuums und Schutz des Gemeinwesens. 's

Zusammengefaßt lautet das Ergebnis des Beitrags von Wilson und Kelling, daß die Verhinderung von Straftaten nur möglich ist, wenn bereits sehr frühzeitig eine umfassende Prävention erfolgt. Ein frühzeitiges und nachdrückliches Eingreifen bereits bei geringfügigen Verstößen gegen Recht und Ordnung wird als entscheidend angesehen. Denn die Duldung gerade auch geringfügiger Verstöße bilde die Grundlage für die Begehung und Verbreitung auch schwererer Straftaten. Auf Grund dieser Annahmen zum Zusammenhang von Ordnung und Kriminalität lehnen Wilson und Kelling nachdrücklich Tendenzen der Entkriminalisierung betreffend geringfügigere Verstöße gegen die Rechtsordnung ab. Gefordert wird vielmehr die Aufrechterhaltung entsprechender Straftatbestände und die systematische Verfolgung gerade auch von geringfügigen Ordnungsverstößen. Insbesondere schließt ihr Konzept es ausdrücklich programmatisch ein, daß harte polizeiliche Mittel auch gegen das Vcrhalten von Personen im öffentlichen Raum zur Anwendung kommen, dic Rechte anderer Personen nicht beeinträchtigen. Hier skeptischen Einwänden treten Wilson und Kelling mit folgender Feststellung entgegen, die zugleich das ganze kriminalpolitische Programm in einem Satz zusammenfaßt:

"Einen einzelncn Betrunkenen oder einen einzelnen Landstreicher zu verhaften, der keiner erkenmbaren Person gcschader hat, scheint ungerecht zu scin, und ist es auch in gewisser Weise. Aber nichts gegen einc Anzahl Betrunkener oder hundert Landstreicher zu untcrnchmen, kann eine ganze Gemeinde zcrstoren. «'t

Auf Grund der besonderen Bedeutung von geringfügigen Ordnungsverstößen bzw. noch weit davor liegenden Vorstufen für ihr Konzept öffentlicher Sicherheit stehen bei Wilson und Kelling neben Vandalismus, Lärmbeeinträchtigungen usw. vor allem Bettler und Alkoholkonsumenten im öffentlichen Raum im Mittelpunkt der Überlegungen. Daß deren Vergehen in der Regel kaum rechtliche Bedeutung haben, ist auch Wilson und Kelling bewußt. Neben möglichen Problemen beim massierten Auftreten entsprechender Personengruppen im Einzelfall, verweisen sie auf das nach ihrer Auffassung berechtigte Anliegen von Gemeindebewohnern, "unliebsame Personen « auch jenseits konkreter Delikte aus der näheren Umgebung entfernen zu können. In der Ermöglichung der Entfernung von Personen und Personengruppen aus dem öffentlichen Raum bestehe auch die eigentliche praktische Bedeutung von rechtlichen Tatbeständen wie "öffentliche Trunkenheit«, "Landstreicherei« usw.:

"Solche Vorwürfe bestehen nicht, weil die Gesellschaft will, daß Landstreicher oder Bctrunkenc bestraft werden, sondern weil sie will, daß ein Polizeibeamter die rechtlichen Mittel hat, eine unliebsame Person aus ciner Gegend zu entfernen, falls die informellen Mitrel, die Ordnung aufrechtzuerhalten, fehlgeschlagen sind. .:

Nach Auffassung von Wilson und Kelling kommt sozialen Ursachen und Rahmenbedingungen nur cine sehr untergeordnete Rolle bei der Entstehung von Kriminalität zu. Insbesondere Wilson als herausragender Vertreter der new realists bezieht sich hier auf ökonomische Theorien der Kriminalität. ${ }^{6}$ Das bedeutet, daß von einem durchaus rational kalkulierenden Akteur ausgegangen wird, der nach Kosten-/Nut-

13 Ebd., 13sfi.

14 Fbd., 131

is Fibd.

16 Dazu Sack (Fn. 3), 116; Hess (Fn. 3), 182, insbesondere die Theorien des Okonomen Gary S. Becker wurden hier aufgegriffen. 
zenabwägung über Handlung oder Unterlassung entscheidet. Zwar werden soziale Ursachen nicht für völlig unbedeutend erklärt, das kriminalpolitische Programm setzt aber vorrangig auf der Grundlage der ökonomischen Theorie der Kriminalität auf die Erhöhung der Kosten krimineller Handlungen. Durch die Erhöhung der Kosten mittels intensiver Strafverfolgung und hoher Strafen soll die Nachfrage gesteuert werden.

Diescr Ansatz der new realists steht in krassem Gegensatz zum ganz überwiegenden Teil der liberalen Kriminologie und entsprechender kriminalpolitischer Konzeptionen bis in die 6oer Jahre in den USA. ${ }^{7}$ In dieser Entwicklungsphase wurden intensiv soziale Ursachen von Kriminalität crforscht und diskutiert. Für alle relevanten kriminalpolitischen Konzeptionen war prägend, daß den Ursachen der Kriminalität vorrangig mit sozialen Maßnahmen begegnet werden muß. Die new realists stellen diese Zusammenhänge in Frage. Die zentrale These lautet: Erfolgreiche Kriminalitätsbekämpfung ist auch ohne Erfassung der sozialen Ursachen oder Rahmenbedingungen der Kriminalität möglich. Vorrangig geht es un eine neue polizeiliche Strategie bei der Bekämpfung der Kriminalität, nicht um eine Fortsetzung der für weitgehend erfolglos erachteten Befassung mit den sozialen Ursachen der Kriminalität.

\section{Die Theorie der broken windows und das Modell New York}

Den Thesen von Wilson und Kelling liegt eine Mischung aus kriminologischen Erkenntnissen und Hypothesen sowie ein handfestes kriminal- und ordnungspolitisches Programm zu Grunde. Die auch bereits von Sack betonte "Suggestivität der Argumentation, der man sich nur mit einiger Anstrengung entziehen « könne ${ }^{18}$, erwächst aus der spezifischen Mischung aus präziser Problembeschreibung, kriminologischen Forschungsergebnissen und Ideologie. Eine Auscinandersetzung ist vorrangig auf Grund der fragwürdigen kriminalpolitischen Schlußfolgerungen notwendig, denn zunehmend setzt sich auch im kommunalen Bereich in der BRD eine Praxis durch, bei der weit unterhalb der Gefahrenschwelle gegen den Aufenthalt von Personen im öffentlichen Raum vorgegangen wird.

Der spezifische kriminologische Ertrag des Beitrags von Wilson und Kelling bedarf einer differenzierten Beurteilung. Behandelt werden von Wilson und Kelling durchaus relevante Probleme des innerstädtischen Raums. Die Grundannahme eines gewissen Zusammenhangs von Unordnung, Verwahrlosung und Kriminalität ist kaum strittig. ${ }^{19}$ Dies bedeutet aber nicht, daß den weitreichenden Schlußfolgerungen von Wilson und Kelling zuzustimmen ist. Vielmehr sind diverse Schlußfolgerungen nicht nur äußerst spekulativ, sondern z. T. auch abwegig. Dies gilt insbesondere für das folgende Beispiel, das die zentrale Grundthese untermauern soll:

„Wenn Bürger nicht einmal einen lästigen Bettler davon abhalten können, Passanten zu belästigen, wird der Dieb meinen, es sei sogar nocb unwahrscheinlicher, daß sie die Polizei rufen werden, um einen potentiellen Straßenräuber zu identifizieren, oder daß sie bei einem Überfall selbst eingreifen werden. ${ }^{20}$

i) Sack (Fn. 3), 117; Hess (Fn 3), 134

18 S.ick (Fn. 3), 119.

$19 \mathrm{~V}$, l. zu der kriminologischen Ursachentheorie der sozialen Desorganisacion allgemein Schneider, Kriminalistik s/1997, $306 \mathrm{f}$.

20 Wilson/Kelling (['n, 2), 128. 
$\mathrm{Daß}$ der ungehinderte Bettler das erste "zerbrochene Fenster « darstellen soll, ist nicht nur rechtlich fragwürdig, sondern entbehrt auch empirisch jeder Grundlage. Natürlich können bestimmte Erscheinungsformen des Bettelns und des Alkoholkonsums usw. eine Verwahrlosung des öffentlichen Raums und die Herausbildung kriminogener Strukturen signalisieren. Betteln und Alkoholkonsum aber allgemein als signifikante Zeichen einer solchen Entwicklung zu betrachten, hält einer seriösen kriminologischen Analyse nicht statt. Hier schlägt bei Wilson und Kelling eine empirisch in keiner Weise abgesicherte, spezifische kriminal- und ordnungspolitische Ideologie durch. " Seincr vermeintlich gesicherten kriminologischen Grundlagen beraubt, bleibt als schlichter Kern übrig: Unerwünschte Personen wie Bettler und Alkohol konsumierende Personen können nach den jeweiligen Vorstellungen einer Gemeinde aus dem öffentlichen Raum entfernt werden.

Andere als polizeiliche Modelle ciner Sicherung des sozialen Raums für alle werden von Wilson und Kelling zwar durchaus angesprochen, aber nicht näher erörtert. So verweisen sic darauf, daß z. B. für die Nutzung von Örtlichkciten eine gütliche Regelung zwischen allen Nutzungsinteressenten (z. B. Jugendliche/Erwachsene) angestrebt werden könne. Derartige Ansätze werden aber von Wilson und Kelling nicht weiterverfolgt. Vielmehr gehen sie überwiegend davon aus, daß alternative Präventionsmodellc nicht erfolgreich gewesen seien. Im Ergebnis plädieren Wilson und Kelling somit für einen Vorrang polizeilicher Prävention. Dieses Argument überzeugt allerdings auch deshalb nicht, da - wic zutreffend beschrieben - auch die polizeilichen Konzepte in der Vergangenheit erhebliche Defizite aufgewiesen hatten, und auch hicr gerade umfassende neue Konzeptionen erprobt wurden.

Der kriminologische und kriminalpolitische Paradigmenwechsel, den die Thesen von Wilson und Kelling beinhalten, fand in der breiten Öffentlichkeit erst mit der praktischen Umsetzung in cinzelnen größeren Modellversuchen Aufmerksamkeit. Während die von Wilson und Kelling in ihrem Beitrag beschriebene Entwicklung im Staat New Jersey zunächst nur begrenzte Aufmerksamkcit fand, hat sich dies vor allem seit der Diskussion über das Modell New York geändert. Das Modell New York steht nicht zufällig im Mittelpunkt des Interesses. Seit viclen Jahren versuchte Rudolph Giuliani mit einer rigorosen law and order campagne die Bürgermeisterwahlen in New York zu gewinnen. Mit seiner Wahl zum Bürgermcister 1993 setzte Giuliani umgehend William Bratton zum Polizeipräsidenten ein. ${ }^{22}$ William Bratton wurde als Transit-Police-Commissioner in New York seit 1990 bekannt. In dieser Funktion begann er seinen Kampf für eine sichere U-Bahn. Die prakrische Polizeiarbeit von Giuliani und Bratton orienticrte sich explizit an den Thesen der new realists. Dabei wurde allerdings eine noch weiter vereinfachte Sichtweise zu Grunde gelegt, die in dieser Schlichtheit selbst von den new realists nicht vertreten wird. Danach existiert zwischen Kriminalität und sozialen Rahmenbedingungen kcinerlei Zusammenhang:

"There has never been a proven connection between the state of the economy and crime, and there is absolutely no correlation betwcen unemployment and crime. " $^{23}$

In diescm extrem schlichten Modell geht es nur noch um die Optimierung der polizeilichen Handlungsformen im Rahmen einer weit ausgreifenden präventiven Strate-

21 Nicht zufallig erschien der Beitrag von Wilson und Kelling erstmals auch keneswegs in einer kriminologischen Fachzettschrift, sondern in Arlantic Monthly, eituer konservativen Monatsschrift, wie auch von Sack vermerkt, Sack (Fn. 3), 119.

22 Dazu Hess (Fn. 3), r8 8 f.; Spiegel $28 / 1997,48 f f$; 1996 wurde Bratton wegen Auseinanderseuzungen z.wischen Giuliani und Bratton becrcffend die Urheberschaic an der Idee des n Aufraumens " von Howard Safir abgelost, Bratton ist jeczt im privaten Sicizerhestsbusincss tatıg.

23 Zitat Giuliani, zituert nach Hess $($ Fn, 3), 382 
gie. Andere als polizeiliche Strategien werden weitgehend ausgeblendet. Diese Auffassung bewegt sich nicht mehr im Theorien- und Meinungsspektrum der seriösen kriminologischen und kriminalpolitischen Debatte. Um so erstaunlicher ist die Aufmerksamkeit, die dem Modell Now York in der neueren Debatte in der BRD zukommt.

$\mathrm{Zu}$ den bis heute in der BRD noch weit verbreiteten essentials der liberalen kriminologischen und kriminalpolitischen Tradition gehört die Erkenntnis von den deutlichen Grenzen polizeilichen Handelns bei der Herstellung von Sicherheit. Kube ${ }^{24}$ unterscheider drei unterschiedliche Präventionsbereiche, ausgehend von primären, sekundären und tertiären Ursachen für Kriminaliät. Primäre Prävention soll die tieferliegenden Ursachen für kriminelles Handeln beseirigen (Abbau von Sozialisationsdefiziten, soziostrukturellen Mängellagen, Stabilisierung des Rechtsbewußtseins). Bezugsfelder sind hier maßgeblich die Bereiche Elternhaus, Freizeit, Ausbildung und Arbeit. Dicser Bereich liegt weitgehend außerhalb der polizeilichen Arbeit. Sekundäre Prävention setzt auf die Reduzierung von Tatgelegenheiten. Dazu gehören Sicherungsmaßnahmen für gefährdete Personen, Sachen und Bereiche. Das Spektrum reicht von technischen Maßnahmen bis zu Verhaltensratschlägen. Nur ein Teil dieser sekundären Prävention kann von der Polizei wahrgenommen werden. Sekundäre Prävention erschöpft sich aber niclut in polizeilicher Arbeit. Sie ist cine gesamtgesellschaftliche Aufgabe, insbesondere auch in kommunalen Bereich. Tertiäre Prävention setzt auf Rückfallvermeidung durch adäquate Sanktionen und Maßnahmen der Resozialisierung. Sanktionen sind dabei streng an den Zielen der Rückfallvermeidung und Resozialisierung orientiert. Auch im Bereich der tertiären Prävention werden entscheidende Beiträge nicht von der Polizei geleistet. ${ }^{25} \mathrm{Das}$ allein an polizeilichen Strategien orientiertc Modell Ncw York blendet diese notwendigen Differenzierungen völlig aus. Eine aufgeklärte Kriminalpolitik kann dagegen auf eine solche differenzierte Sicht nicht verzichten. Dies bedeutet aber, daß Prävention als ein größeres Nctz unterschicdlicher Handlungsfelder und Beteiligter verstanden werden muß, wie es etwa Kube in seinem Systematisierungsversuch dargelegt hat.

Kritischen Einwänden gegen das neue Repressionsmodell begegnen dessen Befürworter mit dem Hinweis auf die Erfolge dieses Modclls. Als typischer Erfolgsmaßstab wird hier in der Regel auf die Kriminalitätsstatistik abgestellt. Das Modell New York liefert hier zweifelsfrei beeindruckendc Daten, die einen relevanten Abbau der Kriminalität in den letzten Jahren ausweisen. ${ }^{26}$ Die immer wieder behauptete Erfolgsquote des Konzepts der Prävention durch Repression ist nach den vorlicgenden Erfahrungen und Untersuchungen aber deutlich in Frage zu stellen. Ein besonders intensiv diskutierter Einwand geht davon aus, daß die Verbrechenslawine von besonders geburtenstarken Jahrgängen ausgelöst worden sci. Der Kriminologe Fyfe, der 16 Jahre bei der New Yorker Polizei tätig war, sieht in der derzeitigen Kriminalitätsentwicklung vor allem cin Ergebnis der abgesteckten Drogenmärkte, auf denen derzeit kcine gravierenden Konkurrenzkämpfe stattfänden. Ein spezifischer Erfolg der besondcren New Yorker Polizeistrategie ist für ihn nicht erkennbar. ${ }^{27}$ Auch Pfeiffer, Direktor des Kriminologischen Forschungsinstituts in Niedersachsen, verwcist auf vielfältige Ursachen des New Yorker Modells: Deutlich verbesserte Notfallversorgung, demographische Entwicklung mit steigendem Altersdurchschnitt

24 Kube, Systematusche Kriminalpravention, Wiesbaden 1986, S. 1of.

25 Kritusch gegenuber polizeilichen Konzeptionen und zu den Alternatıven, Hassemer, Perspektiven einer

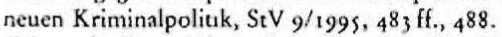

$26 \mathrm{Vgl}$. zu den hier nicht weiter relevanten Einzeldaten Hess (Fin. 3), 186 ff.; Spiegel 28/1997, 48 ff., 53.

27 Zitiert nach M.Schwelien, Das Wunder von New York, DIE ZIiIT v. 24. 3. 1997, S. 7. 
und zurückgegangener Bedeutung der besonders kriminalitätsanfälligen Gruppe der 18- bis z sjährigen, insbesondere aber die Fntstehung von 6 Millionen Jobs seit $1993 .{ }^{28}$

Gegenüber Kriminalitätsstatistiken können bekanntlich eine Vielzahl von Fragen und Einwänden formuliert werden. Hess hat sich eingehend mit verschiedenen Detaileinwänden gegen die New Yorker Erfolgsbilanz auseinandergesetzt, die zwar teilweise berechtigt sind, aber nach Hess die Erfolgsbilanz des Modells New York nicht pauschal in Frage stellen können. ${ }^{29}$ Auch wenn nicht alle sich in der Kriminalitätsstatistik niederschlagenden Daten als Erfolg der neuen polizeilichen Strategie verbucht werden können, bleibt ein relevanter Frfolgskern bestehen. Fntscheidend ist aber letztlich nicht allein, ob durch die repressive Strategic kurzfristig gewisse Erfolge erzielt werden konnten. Über den Erfolg kann gerade bei extrem repressiven Modellen erst im Rahmen von Langzeitstudien ein seriöses Urteil abgegeben werden. ${ }^{30}$

Kritiker des neuen Repressionsmodells in den USA verweisen in diesem Zusammenhang darauf, daß dic Strategie der Kriminalisierung zunehmend größerer Teile der Bevölkerung sich als die eigentliche Zeitbombe für die Zukunfr der USA entwickeln könnte. Loic J. D. Waquandt hat die neuere Entwicklung des repressiven Staatshandelns in den USA näher untersucht. ${ }^{3 t}$ Die sich hier entwickelnde systematische Ersetzung des sozialen Netzes durch Disziplinierung und Kriminalisierung hat bereits heute ein hochgradiges Ausmaß erreicht. Ein wesentliches Element der neuen Repressionspolitik stellt der massive und systematische Rückgriff auf die Institution des Gefängnisses dar. Nach einem Rückgang der Zahl der Häftlinge um ${ }_{2} \%$ in den $60 e r$ Jahren ist dic Zahl von 1970 bis I 991 von knapp 200000 auf beinahe 825000 angestiegen. Waquandt bezeichnet diesen Anstieg um über $300 \%$ in 20 Jahren sicherlich zu Recht als einzigartige Explosion der Häftlingszahlen in einem demokratischen Land. Diese Masseneinsperrung ist in großen Tcilen eine Randgruppeneinsperrung. $56 \%$ der Gefängnisinsassen in den USA entfielen 199 I auf Drogendelikre. Insbesondere die vielen kleinen Dealer in den Armutsvierteln werden besonders häufig von der hier präsenten Polizei aufgegriffen. ${ }^{32}$ Offiziellc Stellen in Washington gehen bei der Berechnung der Kosten für das Gefängniswesen davon aus, daß die Zahl der Inhaftierten in Bundesgefängnissen in den nächsten Io Jahren weiter von 925000 auf 2,26 Millionen steigen wird. ${ }^{33}$

Daneben kommt der neuen Form des Verwahrens von Jugendlichen eine wachsende Bedeutung zu. 59 der 77 größten Städte der USA haben bereits eine nächtliche Ausgangssperre für Jugendliche verhängr. So gilt $z$. B. in Chicago eine generelle nächtliche Ausgangssperre für Jugendliche unter 16 Jahren zwischen 22.30 Uhr (23.30 Uhr am Wochenende) und 6.00 Uhr morgens, soweit kein triftiger Grund vorliegt. Bei Verstößen wird in relevantem Umfang das Wegsperren von Jugendlichen praktiziert. I 992 wurden auf diese Weise bereits 75000 Jugendliche aufgegriffen. ${ }^{34}$

Diese Praxis des Wegsperrens führt zu einer enormen Kostenbelastung (1990 bereits 28 Milliarden Dollar). Dics begründet wiederum nachhaltige Anstrengungen betreffend die Kostenreduzierung. Dazu dienen insbesondere die Privatisierung des

28 Stellungnahme zur Debatte uber das Modell New York in der BRD, in: Frankfurter Rundschau v, 21. 7. 1997 .

29 Hess (Fn. 3), 186

30 So auch Pfeiffer (Fn, 28).

31 L. J. D. Waquandt, Vom wohltatigen Staat zum strafenden Staat, Leviathan 1/r997, soff., insbesondere Abschnite III: Die Kriminalisierung des Elends oder der Vormarsch des strafenden Staates, $57 \mathrm{ff}$.

32 Daten nach Waquandt (Fn. 31), 58.

33 Ebd., 60

34 Ebd. 
Gefängniswesens, aber auch der Abbau von Beschäftigungs- und Ausbildungsprogrammen. Insgesamt entwickelt sich hier eine Spirale der verstärkten Kriminalisierung, an die sich ein perspektivloses Ein- und Wegsperren anschließt. Waquandt kommt deshalb zu dem Schluß:

"Die Expansion des strafenden Staates in den USA ist also keine Reaktion auf die steigende Kriminalität, sondern antwortet auf die sozialen Verwerfungen, die der Rückzug des wohltätigen Staates nach sich gezogen hat. Der Vormarsch des strafenden Staates schafft sich seine eigene Rechtfertigung in dem Maße, wie seine Politik eben jene Zunahme der Kriminalität hervorbringt, der sie doch cincn Riegcl vorschicben sollte. "3s

Angesichts des rigiden Abbaues sozialer Leistungen in den USA prognostiziert Waquant wachsende Unsicherheit und Kriminalität (bis hin zu bürgerkriegsähnlichen Zuständen). In dicsem Sinne formuliert auch Hess seine nachhaltige Skepsis gegenüber den Erfolgen des Modells New York:

"Wie auch immer man die neue New Yorker Polizeistrategic cinschätzr, wenn sie allein zum Vorbild einer künftigen Kriminalpolitik wird, ohne daß man zugleich die prognostizierte Situation durch energische Eingriffe an den root causes entschärft, werden in Zukunft mehr Fensterscheiben zu Bruch gehen denn zuvor. «t"

Ähnlich lautet die Stellungnahme von Pfeiffer. Auch Pfeiffer kann im Modell New York nur sehr begrenzte und allenfalls kurzfristige Erfolge erkennen, die sich angesichts der Folgeprobleme der perspektivlosen Inhaftierungspolitik und neuer Probleme als Pyrrhussieg erweisen könnte. Längerfristige Erfolge seien nur durch eine neue Drogenpolitik und eine Bekämpfung der sozialen Ausgrenzung zu erreichen. So wie bei der Bewcrtung der New Yorker Erfolgszahlen betreftend die Kriminalitätsrate neben sonstigen Faktoren vor allem die verbesserte Arbeitsmarktsituation zu berücksichtigen sei, gelte erst recht für die langfristigen Perspektiven, daß vorrangig der sozialen Ausgrenzung begegnet werden müsse. ${ }^{37}$

\section{Das Modell New York und der Rechtsstaat}

Die alle Grundsätze des tradierten Rechtsstaats weitgehend außer Kraft sctzcndc polizeiliche Praxis bildet das eigentliche Kernstück des Modells New York. An Stelle überkommener Vorstellungen der Bindung an Recht und Gesetz wird der Bewegungsfreiheit der Polizei der absolute Vorrang eingeräumt. Dies bedeutet, daß die Bindung der Polizei an gesetzliche Regelungen weitestgehend gelockert wird. Auch abgelegenstc rechtliche Regelungen wurden herangezogen, um das weit ausgreifende polizciliche Handeln in irgendeincr Weise noch rechtlich begründen zu können. Auf diese Weisc schaffte sich die Polizei weitgehend ihr eigencs Recht. Fast völlig bedcutungslos sind in der polizeilichen Praxis des New Yorker Modells auch Übcrlegungen im Sinne des Grundsatzes der Verhältnismäßigkeit. Dies folgt bereits aus dem Leitprogramm, das ein rigides Vorgehen gerade auch gegen geringfügige Verstößc vorsicht. Dementsprechend sind u. a. erkennungsdienstliche Maßnahmen, Durchsuchungen, Festnahmen und Befragungen auch bei Verstößen gegen Verbote des Alkoholkonsums und des Bettelns durchaus üblich.

Die Kriminalisierung und rigide Verfolgung gerade auch nur geringfügiger Verstöße entsprechend dem New Yorker Modell ist nach der bundesdeutschen Rechtsord-

35 Ebd., 6I.

36 Hess (Fn. 3), 190

37 Pfeiffer (Fn, 28). 
nung evident unzulässig. Darauf weist - bei aller Sympathie für Teile des Modells New York - etwa auch der Frankfurter Polizeipräsident Hoffmann hin. Die New Yorker Polizeipraxis sei »bei uns rechtlich nicht möglich«, im übrigen sei aber auch zweifelhaft, ob eine derartige polizeiliche Praxis in der BRD wünschenswert wäre. ${ }^{38}$

Neben der spezifischen polizeilichen Praxis sind aber auch zahlrciche Verbotstatbestände im kommunalen Bereich in den USA fragwürdig. Insbesondere die in den USA zahlreichen Verbote des Alkoholkonsums und des Bettelns im öffentlichen Raum sind nach der überwiegenden bundesdeutschen Rechtsauffassung nicht haltbar. Hier werden Verbote unterhalb der Gefahrenschwelle erlassen, was nach dem bundesdeutschen Polizei- und Ordnungsrecht unzulässig ist. Im übrigen verstoßen derartige Verbote aber auch gegen Art. 2 I GG, da sie die allgemeine Handlungsfrciheit ohne hinreichenden Grund beschränken. ${ }^{39}$

In der neueren kommunalen Praxis in der BRD verstärken sich allerdings die Versuche, u. a. Verbote des Alkoholkonsums im öffentlichen Raum und Bettelverbote zu erlassen. In dieser kommunalen Praxis entwickelt sich verstärkt eine teilweise dcm New Yorker Modell vergleichbare Konzeption. Zur Begründung derartiger Verbote wird zum Teil explizit auf das New Yorker Modell Bezug genommen, wie etwa durch den Berliner Innensenator Schönbohm. Auch wird in einzelnen bundesdeutschen Großstädten die gezielte Verdrängung unerwünschter Personengruppen aus dem öffentlichen Raum entsprechend dem New Yorker Modell bereits praktiziert.

Vor dem Hintergrund dieser Entwicklung bedürfen die neuen Konzepte in der BRD, die auf dic Herstellung von "Ordnung « im innerstädtischen öffentlichen Raum abzielen, einer besonders kritischen Prüfung. Deutlich zu formulieren sind dic rechtsstaatlichen und verfassungsrechtlichen Grenzen. Wann die Ordnung im öffentlichen Raum aus den Fugen gerät, ist eine Wertungsfrage, bei der insbesondere auch Grundrechte aller Nurzer des öffentlichen Raums zu beachten sind. Keinesfalls zulässig ist hier cine von den Grundrechten losgelöste autonome Entscheidung der Kommunen über das Verhalten von Personen im öffentlichen Raum.

Die hier bestehenden eindeutigen verfassungsrechtlichen Grenzen können auch nicht durch fragwürdige kriminologische Erkenntnisse entsprechend der Theorie der broken windows überwunden werden. ${ }^{40}$ Vereinfachte Betrachtungen von »Unordnung a als Kriminalitätsursache und die daraus vermeintlich erwachsende Rechtfertigung rigiden Vorgehens gerade auch gegenüber nur geringfügigen Ordnungsverstößen finden aber in der BRD zunehmend Anhänger. Hier zeichnet sich derzeit ein massiver Rückfall in die Zeiten vor der Strafrechtsreform ab. Im Zuge der Strafrechtsreform seit Anfang der 7oer Jahre wurdc u. a. der Tatbestand der Betrelei und der Landstreicherei im StGB gestrichen. Zwar blieb nach der Entkriminalisierung derartiger Handlungs- und Lebensweisen immer noch Raun für einen Zugriff der Polizei- und Ordnungsbehörden. Betteln, Alkoholkonsum und Umberzichen wurden aber überwicgend auch nicht mehr als Verstöße gegen die öffentliche Sicherheit

38 Gesprach mit dem Frankfurter Polizeiprasidenten W. Hoffmann, in: Frankfurter Rundschau v. 17.7. 1997.

39 E. Denninger, Polizeiaufgaben, in: F. Denninger/H. Lisken (Hrsg.), Handbuch des Polizerechts, 2. Aufl., Munchen 1996, S. 14 I f.; ders., Polizeirecht, in: Meyer/Stolless (Hrsg.), Hessisches Staats- und Verswaltungsrecht, 4. Aufl., Frankfurt 1996, S. 287; cingehend dazu M. Stolleis/W. Kohl, Rechtsgutachten uber die Zulassigkeit ordnungsrechtlicher Maßnahmen gegen Nichtseßhafte in den Stadten, insbesondere durch Alkoholverbote auf Grund straßenrechtlicher Sondernutzungssatzungen, und die überarbettete Fassung von W. Kohl, NVwZ 199x, 620ff.; W. Hecker, Die Regelung des Aufenthalts von Personen im offentlichen Raum, Darmstadt 1997.

40 Zur grundrechtsorientierten Kriminologie und Krıminalpolıtuk vgl. W. Hassemer, Vortrag zur Eroffnung des 19. Strafverteidigertages in Hamburg 1995, Frankfurter Rundschau v. 25. 3. 1995, S. 6. 
und Ordnung verfolgt. Lediglich in Einzelfällen damic verbundene unzuläsșige Handlungen waren Anlaß für ein Einschreiten von Polizei- und Ordnungsbehörden. Auch die Literatur zum Polizei- und Ordnungsrecht vertrat - ungeachtct einzelner Differenzen zur Bedeutung der "öffentlichen Ordnung « - ganz überwiegend dieses liberal-rechtsstaatliche Konzept. Einen extrem anderen Akzent hat im vorliegenden Zusammenhang bislang lediglich Götz gesetzt. Bereits seit längerem thematisiert Götz »nicht mchr gemeinverträgliches Handcln - wie Bettelei, Trinkgclage, Lärm " im öffentlichen Raum:

*Zahireiche Verhaltensweisen auf offentlichen Straßen, vor allem in den Fußgängerbereichen der Innenstädre werfen die Frage auf, ob sie noch gemeinverträglich sind oder als nicht mebr gemeinverträglich verboten werden sollten: Betteln, insbesondere das aggressive Betteln, das Niederlassen zum Alkohol- oder Drogenkonsum, das Verrichten der Notdurft, der Daueraufenthalt von Stadtstreichern, Ansammlungen von Hunden, Verunrcinigungen durch Abfälle und anderes mehr. « $^{4}$

In der gesamten bundesdeutschen Literatur zum Polizei- und Ordnungsrecht stellt diese pauschale und undifferenzierte Zusanmenstellung verschiedenster Verhaltensweisen bislang eine absolute Ausnahme dar. Dagegen sind in der kommunalen Praxis in den letzten Jahren bereits in zahlreichen Städten in der BRD kommunale Verordnungen und Satzungen erlassen worden, die gezielt Verbotstatbestände zum Alkoholkonsum und Betteln unterhalb der Gefahrenschwelle vorsehen. ${ }^{42}$

\section{Perspektiven}

In der Diskussion über neue Sicherheitskonzepte im kommunalen Raum zeichnet sich eine Entwicklung ab, in der der alte Gegensatz von harter und wcicher Linie in der Kriminalitätsbekämpfung zwar nicht ersetzt, aber durch neue Ansätze ergänzt wird. Konsequente Ahndung von Straftaten und eine verstärkte Prävention muß nicht mit der Ausblendung der sozialen Ursachen der Kriminalität und einer vereinseitigten polizeilichen Strategic einhergehen. Konsequente Ahndung von Straftaten ist auch möglich auf der Grundlage einer differenzierten kriminalpolitischen Konzeption, dic die Bedeutung des Strafrechts für einzelne Tatbestände betont, daneben aber auch offen ist für die Herausnahme geeigneter Tatbestände aus dem Katalog der Straftaten ${ }^{43}$ und für Zurückhaltung bei der Anwendung des Strafrechts, wie z. B. im Bereich des Drogenkonsums ${ }^{14}$, plädiert. Eine solche kriminalpolitische Konzeption steht in striktem Gegensatz zu dem New Yorker Modell, weist aber durchaus Berührungspunkte bei der Einschätzung der Bedeutung der »Ordnung " des öffentlichen Raums, der kommunalen Verantwortung und der Notwendigkeit von Reaktionon auf Rechtsverstöße auf.4s

Mit dem Beispiel von dem zerbrochenen Fenster wird die Frage der Bedeutung stabiler sozialer Strukturen für die Entwicklung von Kriminalität thematisiert. Es ist praktisch unbestritten, daß stabile soziale Strukturen kriminalitätshemmend wirken. Dies ist allerdings vorrangig kcine Fragc polizeilicher Handlungsstrategien. Entscheidend ist hier vielmehr, wie soziale Strukturen unterstützt und gefördert werden können, die den Ursachen von Kriminalität entgegenwirken. Relevant sind hier sol-

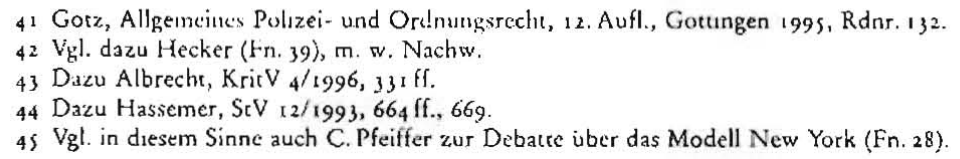


che Strategien, die tiefer an den sozialen Wurzeln der Kriminalität ansetzen. Entsprechende Ansätze der Sozialpolitik, Sozialarbeit und anderer Bereiche existieren seit viclen Jahren. Die Annahme, daß „Ordnung ein vorrangig polizeiliches Handlungsfeld sei, verkennt die vielen Modellversuche z. B. in sozialen Brennpunkten in der BRD, in denen seit Jahren eine Stabilisierung sozialer Beziehungen, Verbcsserungen des Wohnumfelds usw. unter aktiver Mitarbeit der Bcwohncr umgesetzt werden. Die hier angesiedelten Konzepte konkurrieren mit vereinfachten Modellen polizeilichen Handelns. Derart vereinfachte Modelle werden häufig auch wcniger seitens der Polizei vertreten, die sich in weiten Teilen der Grenzen polizcilichen Handelns deutlich bewußt ist, sondern seitens populistischer Politiker, die in zunehmendem Ausmaß gezielt schlichte Handlungsmodelle des „Einschreitens in der Öffentlichkeit vertreten.

In der neueren Diskussion zeichnet sich allerdings ein weitgehender Konsens dahingchend $a b, d a ß$ über die Beseitigung bestimmter Erscheinungen oder bloßer Symbole für Verslumung und Verwahrlosung positive Signale im sozialen Raum gesetzt werden können. Entsprechende Reinigungsaktionen sind in verschiedenen bundesdeutschen Städten bercits angelaufen. Spektakulärc Aktionen richten sich u. a. gegen Graffiti. Auch Wesel ${ }^{46}$ vertritt hierzu die Auffassung, daß die These des Berliner Senats, Verslumung beginne mit Graffiti, teilweise begründet sci. Ähnlich geht auch Pfeiffer davon aus, daß behördenübergrcifende Präventionsmaßnahmen und das Vorgehen gegen "zerbrochene Fenster « optimistische Zeichen setzen können. ${ }^{47} \mathrm{Da}$ durch könne der Wegzug von Bewohnern aus verwahrlosten Gcbieten aufgehalten und das Verantwortlichkeitsgefühl gestärkt werden. Auf der Grundlage dieser Entwicklung sei auch eine allgemeine Stabilisierung der Sozialstruktur mit der Folge einer Abnahme von Straftaten möglich.

Eine deutliche Spannweite tut sich derzeit aber in der Debatte über das richtige Vorgehen auf. Hier wird teilweise mit rigidesten Maßnahmen in Richtung des Modells Ncw York oder Singapur und gezielt auf kommunale Wahlkämpfe ausgerichteten, populistischen Thesen argumentiert. In diesen Konzepten wird vor allem auch auf die gezielte Verdrängung von Problemgruppen aus dem öffentlichen Raum gesetzt. Daneben entwickeln sich aber auch zahlreiche sachliche Ansätze, dic populistische Repressionsmodelle ablehnen, andererseits aber einen ernstzunehmenden Handlungsbedarf im Hinblick auf einzclne Entwicklungen im öffentlichen Raum sehen..$^{8}$

In das Blickfeld kommt hier dic kommunale Verantwortung nicht bloß für das Erscheinungsbild der Städte, sondern eine weitergehende, ncue kommunale Verantwortung für den öffentlichen Raum. Völlig zutreffend werden hicr erhebliche Defizite konstatiert. Wesentliche Teile kommunaler Einrichtungen wurden in vielen Städten dem Verfall preisgegeben. Dabei geht es um öffentliche Plätze und Parks, Toiletten usw. Nicht nur Spritzen von Drogenkonsumenten, sondern auch der Mißbrauch öffentlicher Parks und Kinderspielplätze als Hundeklo durch "normale" Bürger gehören zu dem Problemfeld. Zahlreiche sonstige öffentliche Einrichtungen wurden seitens der Kommunen dem Verfall preisgegeben. Die zu konstatierenden Probleme im Bcreich öffentlicher Einrichtungen erschöpfen sich somit keineswegs allein in bestimmten Ordnungsverstößen von Bürgern. Die aktuelle Diskussion über Graffiti und andere Problembereiche ist deshalb bei aller diskussionswürdigen Pro-

${ }_{46}$ U. Wesel, Nachdenken uber Graffiti, NJW $1997,1965$.

47 Pfeitfer (Fn. 28).

48 Naher zu den in Betracht kommenden rechelichen Maßnahmen gegenuber Graffitı, Wesel (Fn. 46); Spregel 28/1997, ${ }_{4}$ f. zum spezifischen Berliner Modell des rıgiden no zero gegeniber Graffits. 
blematik verkürzt, wenn nicht diese umfassenderen Probleme der Entwicklung des öffentlichen Raums ins Blickfeld geraten.

Die Kommunen tragen zu einem Großteil selbst die Verantwortung für den Verfall von wesentlichen Teilen des öffentlichen Raums. In den Planungskonzepten der vergangenen Jahrzehnte ging die Erschlicßung der Innenstädte in weiten Bcrcichen mit einer sinkenden kommunalen Verantwortung für den allgemeinen öffentlichen Raum jenseits kommerziell genutzter Flächen einher. Mit der wichtigste Aspekt der Debatte über das Modell New York besteht in der Betonung dieser kommunalen Verantwortung für den öffentlichen Raum. Eine aktiverc Wahrnehnung dieser kommunalen Verantwortung ist auch ohne Übcrnahme des spezifischen New Yorker Reprcssionsmodells möglich. Ergänzend zu weitergchenden sozialpolitischen Maßnahmen können als Beispiele für positive Signale im öffontlichen Raum etwa funktionsfähige Sitzmöglichkeiten außerhalb kommerzieller Einrichtungen, Toilettenanlagen usw. "für alle" genannt werden. ${ }^{49}$ Wichtige ncucre Ansätze sind im Rahmen von Projekten erarbeitet worden, in denen es z. B. um das soziale Umfeld von Schulen oder neue Konzeptionen in sozialen Brennpunkten unter aktiver Mitwirkung der Bewohner geht. ${ }^{\circ}$ Ein solchcs Konzept setzt (im Gegensatz zu der aktuellen Tendenz der gezielten Vertreibung einzelner Personengruppen aus $\mathrm{dcm}$ innerstädtischen Raum) auf die Wiedergewinnung eines funkrionsfähigen öffentlichen Raums für alle.

Neben der hier angelegten verstärkten kommunalen Verantwortung für den öffentlichen Raum wird aber auch eine gewisse verstärkte Präsenz von Polizei- und Ordnungsbehörden im öffentlichen Raum weitgehond für sinnvoll und notwendig erachtet. Der soziale Raum ist heute Nutzungsansprüchen unterschicdlichster Nutzer ausgesetzt. Die Ablösung zahlrcicher genereller Verhaltensverbote durch die Zulassung häufig konkurrierender Nutzungen bringt zahlreiche Konflikte mit sich. Hicr ist - neben anzustrebenden sonstigen Nutzungsvereinbarungen und anderen Konfliktlösungen - auch eine verstärkte Präsenz von Polizei- oder Ordnungsbchörden erforderlich, um eine adäquate Nutzung des öffentlichen Raums für alle sicherzustellen. Die Nutzung von Fußgängerzonen, öffentlichen Parks und Anlagen wird seit einigen Jahren bundesweit unter dicsem Gesichtspunkt diskutiert. Auch wenn die spezifischen Handlungsstrategien des New Yorker Modells abzulehnen sind, besteht hier in vielen Fällen ein Handlungsbedarf. Zutreffend ist, daß bei verdichtetem Auftreten auch bloße Belästigungen zu einem Problem werden könncn. Insoweit ist auch Wilson und Kelling zuzustimmen, daß eine "gang" eine Gemeinschaft schwächen kann, auch wenn sie nur in bedrohlicher Weise herumsteht oder Passanten anpöbelt. In der bundesdeutschen Diskussion hat sich auch Sack für cine ernsthafe Debarte über das Modcll New York ausgesprochen. Bei aller Kricik an dem Handlungskonzept im einzelnen unterstreicht Sack zu der Grundsatzfrage:

"Es hilft nichts - die Polizei muß ran. Die haben sich zu lange mit der feinen Aufgabe der Jagd nach dem organisierten Verbrechen beschäftigt, jetze müssen sie sich auch in Deutschland mal um den alltäglichen Kram der Bürger kümmern. «s"

49 Vgl. unsoweir auch die viclzitierten Blumen in offentichen Toıletten im Modell New York.

so Vgl. dazu Egert-Rosenthal, Stadtteilarbeit - Lokate Vernetzung der soztalen und kriminalen Pravention in einum sozialen Brennpunkt, Schrifrenrcihe des Ratcs fur Kriminalitátsverhutung Schleswig-Holstcin, Bd. 6 (1996); in Hessen wird seit vielen Jahren von der Landesarbeitsgeneussthaft Sozıale Brennpunkte (LAG) eine Stadtteilentwicklung in sozialen Brennpunkten unter aktıver Mitwirkung der Bewohner durchgefuhrt.

5I SPIEGEL-Gesprach zur inneren Sicherheit in der BRD und dern Modell New York, DER SPIEGEL 32/1997. $\$ 4 \mathrm{ff} ., 56$. 

"Ob« einer verstärkten Präsenz von Polizei- oder Ordnungsbehörden. Streitig sind allein der Umfang der Umsetzung diescs Präsenzmodells, und vor allem dic dabei umzusetzenden Handlungskonzepte.

Das Modell der Sichcrheit durch mchr Präsenz wurde noch vor wenigen Jahren wesentlich kritischer bcurteilt. So vcrwies etwa Bull in einer deutlich skeptischen Einschätzung vor einigen Jahren auf negative Erfahrungen mit Präsenzkonzepten im Sinne von Zufallsfestnahmen, bloßer örtlicher oder zeitlicher Verdrängung von Kriminalität, aber keiner nachhaltigen Verhütung. ${ }^{52}$ Bull sah denn auch in der Erhöhung des subjektiven Sicherheitsgcfühls der Bevölkerung dic eigentliche Rechtfertigung des Konzepts der Sicherheit durch Präsenz. Von dieser Überlcgung gehen - allerdings unter anderen Vorzeichen - auch Wilson und Kelling aus. In den letzten Jahren werden subjektive Sicherheitsbedürfnisse (auch jenseits objektiver Gefahrenlagen) im kommunalen Raum in der BRD verstärkt berücksichtigt. Soweit sich derartige Maßnahmen auf Reinigungsmaßnahmen, neue Informationseinrichtungen für Bürgerinnen und Bürger und bloße verstärkte Präscnz von Polizei- und Ordnungsbehörden beziehen, erwachsen aus diesen Konzepten (außer den erforderlichen sachlichen und personellen Voraussetzungen) kaum Probleme. Problematisch werden diese Konzepte aber dann, wenn bloße subjektive Sicherheitsbedürfnisse zu nachhaltigen Grundrechtseinschränkungen für andere im Rahmen weit ausgreifender Prävention führen sollen."s Hier bedarf es einer stärkeren Differenzierung. Ernstzunehımenden subjektiven Sicherheitsbedürfnissen von Bürgerinnen und Bürgern kann durch diverse polizeiliche und kommunale Aktivitäten Rechnung getragen werden, ohne daß damit Grundrechtseingriffe bei anderen verbunden sind. Eine Becinträchtigung von Rechten anderer setzt aber voraus, daß auch objektiv eine Gefahrenlage gegeben ist. Auf rein subjektive Sicherheitsbcdürfnisse gestiitzte Grundrechtseingriffe sind unzulässig. Diese im demokratischen Rechtsstaat zentrale Unterscheidung wird von einzelnen neueren kommunalen Handlungskonzepten in Anlehnung an das Modell New York gezielt unterlaufen. Dies gilt insbesondcre für die planmäßige Vertreibung einzeIner Personengruppen unter Verweis auf subjektive Sicherheitsbedürfnisse von Bürgerinnen und Bürgern.

Auch wenn eine erhöhte Präsenz von Polizei- oder Ordnungsbehörden im öffentlichen Raum als erforderlich und sachgerecht angeschen wird, bleibt aber weiter entscheidend, daß Polizei und Ordnungsbchörden gerade im Bereich der Primärprävention an deutliche Grenzen stoßen. Die gesellschaftlich bequeme Zuweisung einer Alleinverantwortung von Polizei, Ordnungsbehörden und Justiz für Kriminalität in der Gesellschaft wird zunehmend in Frage gestellt. Bull hat bereits frühzcitig darauf hingewiesen, daß eine Einbeziehung der Gesellschaft in die Kriminalprävention erforderlich sei. Dabei betont gerade Bull die Aufgaben der Kommunen. Zwar obliege die Kriminalitätsbekämpfung im engeren Sinne vorrangig der Polizei und der Justiz, Kriminalitätsverhütung sei aber auch eine zentrale kommunale Aufgabe:

"... es ist eine ureigene Aufgabe der kommunalen Selbstverwahtung, bei der Gestaltung der örclichen Lebensverhaltnisse auch auf Vermeidung von Kriminalitätsursachen hinzuwirken. «14

52 H.P. Bull, Die Burger mussen sıch an der Krimınalitatsbekampfung beteiligen, Vortrag auf einer Fachtagung zum Thema Kriminalpravention, Frankfurter Rundschau v. 12.1. 1995.

53 Allgemein zur Frage emes Grundrechts auf Sicherheıt kriusch Knıesel, Innere Sicherhest und Grundgesetz, ZRP 1996, 482 ff.; grundlegend zu den Problemen des Praventionsstaats, Denninger, Der Praventions-Staat, KJ 1/1988, iff.; Sack, in: Gossner (Hrsg,), Mythos Sicherheit (1995), S. 429 ff.

$\varsigma_{4}$ H. P. Bull (Fn. 52 ). 
Kriminalitätsverhütung muß danach verstärkt zu einem systematischen Planungsgegenstand der Kommunen wcrden. " Das Handlungsfeld reicht von der Städteplanung, Architektur, Ordnungs- und Sozialpolitik bis zum kulturellen Sektor. Bull verweist in diesem Zusammenhang auf die schon lange bestehenden kommunalen Strategien in einigen anderen europäischen Ländern. Insbesondere sind hier die aus eigencr Initiative seitens der Kommunen in England praktizierten Konzeptionen zu nennen. Die in den letzten Jahren in der BRD zunehmend tätigen Präventionsrätc ${ }^{56}$ und sonstige Arbeitskreise greifen diese neue Perspektive auf. Eine hier ansetzende Kooperation von Kommunen, Polizei und sozialen Trägern dürfte (im Rahmen der begrenzten kommunalen Möglichkeiten) am ehesten erfolgversprechende Konzepte hervorbringen. Abzulehnen sind dagegen solche Varianten der Prävention, in denen die Kommunen und soziale Träger lediglich unter polizeilicher Oberhoheit in die Präventionsarbeit einbezogen werden. ${ }^{57}$ Die hier in der BRD derzeit geführte Debatte über die richtige Präventionsarbeit steht erst am Anfang. ${ }^{s}$ Klingst hat in der ZEIT-Serie zur aktuellen Debatte über eine ncue Sicherheits- und Kriminalpolitik den veränderten Rahmen der Diskussion treffend umrissen:

"Sicherheit verbindet fürsorgliche Sozialarbcit mit robuster, zupackender Prävention; notwendige Resozialisierung von Straftatern mit konsequenter Abndung der Straftaten. Harte oder weiche linie - das ist der alte Gegensacz. Eine moderne Kriminalpolitik, die den heutigen sozialen Veränderungen gerecht werden will, muß darüber hinausdenken. «`y

Bezugnahmen auf das spezifische no zero Konzept New Yorker Spielart sind für diese Debatte sachlich nicht erforderlich und unter rechtlichen Gesichtspunkten nach bundesdeutschom Recht eindeutig unzulässig. Bei der kritischen Betrachtung des neuen Repressionsmodells in den USA ist aber darauf hinzuweisen, daß es gerade in den USA auch seit vielen Jahren cine Vielzahl altcrnativcr, positiver Ansätzc gibt. Nachbarschaftsprojekte, in denen Bewohner mit kommunaler Förderung ihr Wohnviertel neu gestalten, entwickelten sich insbesondere auch in New York seit Mitte der 8 oer Jahre. Erst kürzlich wählte die National Civic League in den USA die Bronx in New York aus dem Kreis von 20 Mitbewerbern als Vorzeigemodell für entsprechende Modellc aus. Sie würdigte damit die in großem Umfang erfolgte Sanierung von Wohnungen, Neugestaltung der sozialen Einrichtungen und des sozialen Raums unter aktiver Mitwirkung von Nachbarschaftsgruppen. Mit diescm Projckt wurden deutliche Fortschritte bei der Neugewinnung von Lebensqualität und Sicherheit in der Bronx erzielt. ${ }^{60}$ Dieses und entsprechend andere Projekte entwickelten sich in den USA zeitgleich zu der alternativen Konzeption des von Wilson und Kelling befürworteten Repressionsmodells. ${ }^{61}$ In der BRD konkurrieren derzeit ebenfalls schlichte Modelle verstärkter Repression und bloßer Kompetenzerweitcrung für Polizei- und Ordnungsbehörden mit einer Vielzahl anderer Modclle unter dem

ss Eingehend dazu Dreher, Die Polize $7 / 96,173$; Jager, Krımunalıtatsverhutung in Kreis und Kommune, in: Schriftenreihe des Rates fur Kruminalitatsverhutung in Schleswig Holsten Bd. 1 (1993), S. 6 ff.; Finkel, Knmınalicatsverhutung als gesamtgesellschaftlıche Aufgabe, in: Gossner (Fn. 53); Stokar, Kriminalpraventionsrate, Burgerrechte und Polizei/CILIP 57 (2/95), 57 ff. zur Entwicklung in Niedersachsen auf der Landesebene und in den Kommunen; Feles (Hrsg.), Kommunaie Kriminalpravention in Baden-Wurttemberg, 1995; Heınz, Krimınalisuk 6/1997, 426 ff.

s6 Vgl. dazu Ammer, Kommunale Krimunalıtátspraventıon - Chancen durch kommunale Rate fur Krimınalitatsverhutung, in: Schriftenreihe des Rates fur Krımınalitatsverhutung in Schleswig-Holsteın Bd. 2.

57 Zur Debatte uber die neue Rolle der Polıze Feltes/Gramckow, Neue Krimmalpoltik 3/1994, $16 \mathrm{ff}$.; Feltes, Die Polize1 8/1995, $233 \mathrm{ff}$; Dreher (Fn. $55,175 \mathrm{ff}$.); Stokar (Fn. 55), 61 ff.

$58 \mathrm{Vgl}$. die instruktive Darstellung der konzeptionellen und praktischen Unterschiede der eınzelnen Modelle kommunaler Praventionsrate im Spıegel 28/1997, $58 \mathrm{ff}$.

59 M. Klingst, Zeigen, wo die Grenzen sind, DIE ZEIT r3/1997 v. 21. 3. 1997.

60 Frankfurter Rundschau v. 28.8. 1997.

61 Zur entsprechenden Entwicklung in England, Scraton, Burgerrechte und Polize1/CILIP 52 (3/95), 75. 
gemeinsamen Oberbegriff neuer kommunaler Präventionspolitik. ${ }^{62}$ Trotz der zunehmend stärker zu vernehmenden populisrischen law and order Kampagnen bestehen weiterhin Chancen für alternative Konzepte. Denn das Bewußtsein sozialer Verantwortung der Kommunen ist - auch über dic BRD hinaus - in den europäischen Stadtverwaltungen und der Einwohnerschaft insgesamt weiterhin relativ stark verankert. ${ }^{63}$

Joachim Weber

\section{Zum Genugtuungsinteresse des Verletzten als Strafzweck}

Gegenstand der vorliegenden Untersuchung ist das Genugtuungsinteresse des Verletzten als Strafzweck. Der Erste Teil der Arbeit definiert strafrechtliche Genugtuung dogmatisch als Ausgleich des intellektuellen Verbrechensschadens durch Strafe. Der Zweite Teil der Studie untersucht die Dogmengeschichte der Strafzwecke unter besonderer Berücksichtigung der Genugtuung des Verletzten, während der Dritte Teil das Genugtuungsinteresse des Verletzten in Normierungen des Zivilrechts, Strafrechts und Strafverfahrensrechts analysiert. Im Vierten Teil entwickelt der Verfasser ein die Genugtuung des Verletzten berücksichtigendes Strafzumessungsmodell. Der Fünfte Teil der Studie enthält eine Zusammenfassung der Untersuchung in Thesen.

1997, J8I S., brosch., 69, - DM, 504,- öS, 62,50 sFr,

ISBN 3-7890-4849-6

(Nomos Universitätsschriften - Recht, Bd. 258)

NOMOS Verlagsgesellschaft

76520 Baden-Baden 